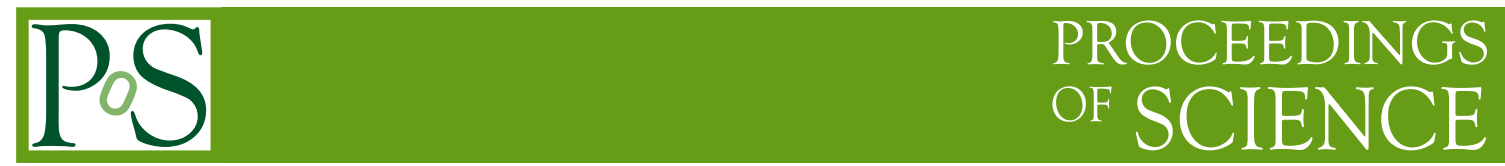

\title{
The Witten Index Revisited
}

\section{Pierre van Baal*}

Instituut-Lorentz for Theoretical Physics

University of Leiden, P.O.Box 9506

NL-2300 RA Leiden, The Netherlands

E-mail: vanbaal@lorentz.leidenuniv.nl

We attempt to deal with the orbifold singularities in the moduli space of flat connections for supersymmetric gauge theories on the torus. The fields are restricted to the fundamental domain, containing no gauge copies, but requiring a boundary condition in field space.

LIGHT CONE 2008 Relativistic Nuclear and Particle Physics

July 7-11 2008

Mulhouse, France

${ }^{*}$ Speaker. 


\section{Introduction}

We revisit supersymmetric Yang-Mills theories on the torus to study the vacuum state in connection with the Witten index[1]. The torus geometry is crucial to preserve the supersymmetry. The index counts the number of quantum states (fermionic states with a negative sign). Due to supersymmetry, states at non-zero energy occur in fermionic and bosonic pairs, and do not contribute to the Witten index. The counting can therefore be reduced to the vacuum sector.

Witten argued that the wave function is constant on the moduli space of flat connections and the degeneracy due to the constant abelian gluino components costs no energy [1]. Requiring gauge invariance (under Weyl reflections) one has $r+1$ ( $=2$ for $\mathrm{SU}(2)$ ) invariant vacuum states which are all bosonic, $|0>, U| 0>, \cdots, U^{r} \mid 0>$, where $U=\sum_{a=1}^{r} \bar{\lambda}_{\dot{\alpha}}^{a} \bar{\lambda}_{\dot{\beta}}^{a} \varepsilon^{\dot{\alpha} \dot{\beta}}$. Untill 1998 there was a puzzle: It didn't agree with the infinite volume gluino condensate calculations for $\mathrm{SO}(\mathrm{N} \geq 7)$ and the exceptional groups[2]. (Additional references are contained in [3].) Witten solved that by finding an extra disconnected component in the moduli space for $\mathrm{SO}(7)$, using a D-brane orientifold construction [4]. This means that their are isolated points for $\mathrm{SO}(7)$ and $\mathrm{SO}(8)$ and for $\mathrm{SO}(\mathrm{N} \geq 9)$ there is an extra component which is like the trivial component for $\mathrm{SO}(\mathrm{N}-7)$. One therefore finds $1+r(S O(N))+1+r(S O(N-7))=h(S O(N))$, or $1+[\mathrm{N} / 2]+1+[(\mathrm{N}-7) / 2]=\mathrm{N}-2(h$ is the dual Coxeter number). This is precisely the infinite volume result! The field theory construction works also for

\begin{tabular}{|c|c|cccccc|}
\hline Group & & \multicolumn{7}{|c|}{ Vacuum-type } & 6 \\
$G$ & $h$ & 1 & 2 & 3 & 4 & 5 & 6 \\
\hline$S U(N)$ & $N$ & $N$ & & & & & \\
$S p(N)$ & $N+1$ & $N+1$ & & & & & \\
$S O(2 N+1)$ & $2 N-1$ & $N+1$ & $N-2$ & & & & \\
$S O(2 N)$ & $2 N-2$ & $N+1$ & $N-3$ & & & & \\
$G_{2}$ & 4 & 3 & 1 & & & & \\
$F_{4}$ & 9 & 5 & 2 & $(1+1)$ & & & \\
$E_{6}$ & 12 & 7 & 3 & $(1+1)$ & & & \\
$E_{7}$ & 18 & 8 & 4 & $(2+2)$ & $(1+1)$ & & \\
$E_{8}$ & 30 & 9 & 5 & $(3+3)$ & $(2+2)$ & $(1+1+1+1)$ & $(1+1)$ \\
\hline
\end{tabular}

Table 1: Contributions to the index for small volumes (A. Keurentjes, JHEP 05, 001(1999))

exceptional groups [5, 6], and is based on classifying triples, $\Omega_{i}=P \exp \left(\int_{0}^{L} A_{i} d x_{i}\right)$, which satisfy $\Omega_{i} \Omega_{j}=\Omega_{j} \Omega_{i}$. Also here one finds that the index in small volumes equals the large volume results.

But a technical problem occurs when using periodic boundary conditions. This is the breakdown of the adiabatic approximation in the reduction of the degrees of freedom to those of the classical vacuum. For SU(2) the classical vacuum is defined up to a gauge transformation by the set of zero-momentum abelian gauge fields, $A_{i}=\frac{i c_{i}^{3} \tau_{3}}{2 L}$, where $c_{i}^{3}$ is constant and $\vec{\tau}$ are the Pauli matrices. Its gauge invariant parametrization is in terms of the Wilson loops that wind around the three compact directions of the torus, which are compact variables. This describes the vacuum valley as an orbifold, $T^{3} / Z_{2}$ for SU(2). Here $g_{[\vec{n}]}=\exp \left(-2 \pi i \vec{n} \cdot \vec{x} \tau_{3} / L\right)$ (which maps $c_{i}^{3}$ to $c_{i}^{3}+4 \pi n_{i}$ ) and $g=i \tau_{2}$ (which maps $c_{i}^{3}$ to $-c_{i}^{3}$ ) give Gribov copies. The orbifold singularities arise where the flat connection is invariant under (part of) the Weyl group (the remnant gauge transformations that 
leave the set of zero-momentum abelian gauge fields invariant). For SU(2) their are eight orbifold singularities (related to $A=0$ by anti-periodic gauge transformations), $c_{i}^{3}=-c_{i}^{3}+4 \pi n_{i}$, giving $c_{i}^{3}=2 \pi n_{i}$ (with $n_{i}=0$ or 1). It was studied in [3] and we will review some of it here.

\section{The Hamiltonian}

We choose the dependence on the bare coupling constant such that

$$
\mathscr{L}=-\frac{1}{4 g_{0}^{2}}\left(F_{\mu \nu}^{a}\right)^{2}+\frac{i}{2 g_{0}^{2}} \bar{\lambda}^{a} \gamma_{\mu}\left(D^{\mu} \lambda\right)^{a} .
$$

The reduction to the zero-momentum degrees of freedom, as in the bosonic case, will replace the bare coupling constant by a running and asymptotically free coupling constant $g(L)$. The zeromomentum gauge fields are parametrized as $A_{i}=i c_{i}^{a} \tau_{a} /(2 L)$. The vacuum valley is parametrized by the abelian degrees of freedom. These are defined by $r_{i}$, with $r_{i} r_{j}=\sum_{a} c_{i}^{a} c_{j}^{a}$, for each $i$ and $j$. As said, we may also parametrize the vacuum valley by $r_{i}=C_{i} \equiv c_{i}^{3}$.

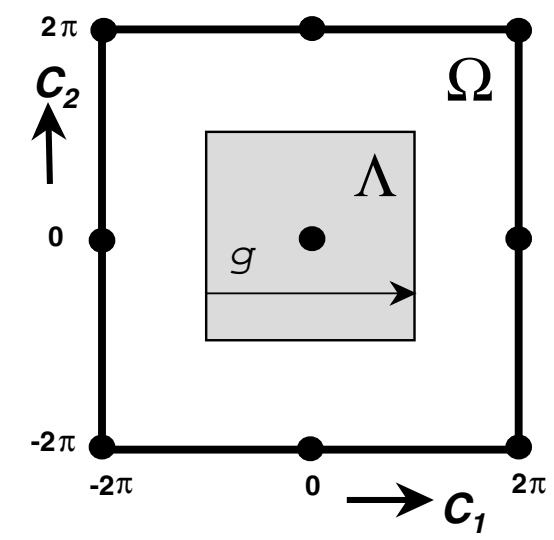

Figure 1: A two dimensional slice of the vacuum valley along the $\left(C_{1}, C_{2}\right)$ plane. The grey square is the fundamental domain $\Lambda$. The dots are gauge copies of the origin (which turn out to lie on the Gribov horizon $\Omega$, indicated by the fat square).

The cell $\vec{C} \in[-\pi, \pi]^{3}$ can be used as a fundamental domain $\Lambda$. Any point on the vacuum valley can be reached by applying suitable gauge transformations. This reduces the eight orbifold singularities to one, but at a cost: Opposite sides on it's boundary are identified by homotopically non-trivial gauge transformations (indicated by $g$ in Fig. 1). The representations of their homotopy define the electric flux quantum numbers as introduced by 't Hooft [7]. We will here only consider the sector with zero electric flux, i.e. the trivial representation, where wave functions at opposite sides are equal. Since the orbifold singularity implies massless excitations for the transverse fluctuations, we seemingly have no choice: We have to keep the non-abelian zero-momentum modes, and thus the adiabatic approximation breaks down.

The energy gap in the fluctuations transverse to the vacuum valley is easily read off. Close to the origin it is given by $2|\vec{C}| / L$. Integrating out transverse degrees of freedom is only reliable if the energy of the low-lying states is smaller than this gap. This energy behaves as $g^{2 / 3}(L) / L$. Consider now a sphere of radius $g^{1 / 3}(L)$ around each orbifold, beyond which the adiabatic approximation is 
accurate. There, the wave function can be reduced to the vacuum valley and, assuming indeed it has zero energy, it will be constant. As long as $g(L)$ is small, we may assume the wave function in the neighborhood of the orbifold singularity to respect spherical symmetry. In the bosonic case, once the wave function spreads out over the vacuum valley, any spherical symmetry is quickly lost. In the supersymmetric case, however, the reduced wave function on the vacuum valley will become constant up to exponential corrections at distances much greater than $g^{2 / 3}(L)$ from the orbifold singularities, which are separated over a distance $2 \pi$. Instead of insisting that the groundstate wave function is normalizable, we should rather insist on its projection to the vacuum valley to become constant. Spherical symmetry near the orbifold singularities will dramatically simplify the analysis.

We will now set up the zero-momentum supersymmetric Hamiltonian, and its reduction to the gauge invariant, spherically symmetric sector. This is not new [8], but we will be able to push it to the point where we can explicitly construct a complete basis of states that respect these symmetries. We start from the supercharge operators,

$$
Q_{\alpha}=\sigma_{\alpha \dot{\beta}}^{j} \bar{\lambda}_{a}^{\dot{\beta}}\left(-i \frac{\partial}{\partial V_{a}^{j}}-i B_{j}^{a}\right), \quad \bar{Q}_{\dot{\alpha}}=\lambda_{a}^{\beta} \sigma_{\beta \dot{\alpha}}^{j}\left(-i \frac{\partial}{\partial V_{j}^{a}}+i B_{j}^{a}\right),
$$

with $V_{i}^{a} \equiv c_{i}^{a} /(g(L) L)$ and $\sigma^{j}=\tau^{j}$ (and $\sigma^{0}$ the unit) as $2 \times 2$ matrices. Restricting to the zeromomentum modes, both the Weyl spinors $\lambda_{a}^{\beta}$ and $\bar{\lambda}_{a}^{\dot{\beta}}$ are constant. Lowering indices is done with $\varepsilon_{\alpha \beta}=\varepsilon_{\dot{\alpha} \dot{\beta}}=-i \tau_{2}, \delta_{a b}$ and $\eta_{\mu \nu}=\operatorname{diag}(1,1,1,-1)$ (or $\delta_{i j}$ ) for respectively the spinor, group and space-time (or space) indices, and raising of indices is done with the inverse of these matrices. Repeated indices are assumed to be summed over, but to keep notations transparent we will not always balance the positions of the gauge and space indices. Finally, for zero-momentum gauge fields one has $B_{i}^{a}=-\frac{1}{2} g \varepsilon_{i j k} \varepsilon_{a b c} V_{j}^{b} V_{k}^{c}$.

In the Hamiltonian formulation the anti-commutation relations $\left\{\lambda^{a \alpha}, \lambda^{b \beta}\right\}=0,\left\{\bar{\lambda}^{a \dot{\alpha}}, \bar{\lambda}^{b \dot{\beta}}\right\}=$ $0,\left\{\lambda^{a \alpha}, \bar{\lambda}^{b \dot{\beta}}\right\}=\bar{\sigma}_{0}^{\dot{\beta} \alpha} \delta^{a b}$, with $\bar{\sigma}_{0}$ the unit $2 \times 2$ matrix (one has $\left(\bar{\sigma}^{\mu}\right)^{\dot{\alpha} \alpha}=\varepsilon^{\dot{\alpha} \dot{\beta}} \varepsilon^{\alpha \beta}\left(\sigma^{\mu}\right)_{\beta \dot{\beta}}$ ), give $\left\{Q_{\alpha}, \bar{Q}_{\dot{\alpha}}\right\}=2\left(\sigma_{0}\right)_{\alpha \dot{\alpha}} \mathscr{H}-2\left(\sigma^{i}\right)_{\alpha \dot{\alpha}} V_{i}^{a} \mathscr{G}_{a}$, where

$$
\mathscr{G}_{a}=i g \varepsilon_{a b c}\left(V_{j}^{c} \frac{\partial}{\partial V_{j}^{b}}-\bar{\lambda}^{b} \bar{\sigma}_{0} \lambda^{c}\right)
$$

is the generator of infinitesimal gauge transformations, and $\mathscr{H}$ is the Hamiltonian density

$$
\mathscr{H}=-\frac{1}{2} \frac{\partial^{2}}{\partial V_{i}^{a} \partial V_{i}^{a}}+\frac{1}{2} B_{i}^{a} B_{i}^{a}-i g \varepsilon_{a b c} \bar{\lambda}^{a} \bar{\sigma}^{j} \lambda^{b} V_{j}^{c}
$$

Splitting the Hamiltonian, $\int d^{3} x \mathscr{H} \equiv g^{2 / 3}(L) H / L$, in its bosonic and fermionic pieces, $H=H_{B}+$ $H_{f}$, we find with $c_{i}^{a}=g^{2 / 3}(L) \hat{c}_{i}^{a}$ and $\hat{B}_{a}^{i}=-\frac{1}{2} \varepsilon^{i j k} \varepsilon_{a b d} \hat{c}_{j}^{b} \hat{c}_{k}^{d}$ that $H_{B}=-\frac{1}{2}\left(\partial / \partial \hat{c}_{i}^{a}\right)^{2}+\frac{1}{2}\left(\hat{B}_{i}^{a}\right)^{2}$ and $H_{f}=-i \varepsilon_{a b d} \bar{\lambda}^{a} \bar{\sigma}^{i} \lambda^{b} \hat{c}_{i}^{d}$.

The orbifold singularities, other than at $\hat{c}=0$, lie at a distance $b=2 \pi g^{-2 / 3}(L)$ in these new variables $\hat{c}$ (measured along the vacuum valley where $\hat{B}$ vanishes). We want to solve for the groundstate wave function such that for $|\hat{c}| \gg 1$ it becomes a constant, after projecting to the vacuum valley. As this boundary condition is compatible with spherical symmetry, i.e. it goes to the same constant for all directions on the vacuum valley, we will restrict ourselves to wave functions $\Psi(\hat{c})$ that are spherically symmetric and gauge invariant. We stress again that this is an accidental spherical symmetry, that holds in sufficiently small volumes. 


\section{Vacuum valley and boundary condition}

States with odd fermion number $F$ do not respect the symmetry and particle-hole duality relates $F=0$ to $F=6$ and $F=2$ to $F=4$ [3]. Without fermions $(F=0)$ this will reduce to the bosonic Hamiltonian which is known to have a non-zero vacuum energy [9]. We therefore write down the most general $F=2$ states, that is the two-spinor states which are symmetric or antisymmetric in the gauge index (and thus respectively antisymmetric and symmetric in the spinor index), $|\mathscr{V}\rangle \equiv \mathscr{V}_{j}^{a} \mathscr{I}_{a}^{j} \equiv-2 i \mathscr{V}_{j}^{c} \varepsilon_{a b c} \bar{\lambda}_{\dot{\alpha}}^{a}\left(\bar{\sigma}^{j 0}\right)_{\dot{\beta}}^{\dot{\alpha}} \bar{\lambda}^{b \dot{\beta}}|0\rangle$ and $|\mathscr{S}\rangle \equiv \mathscr{S}_{a b} \mathscr{J}^{a b} \equiv-\mathscr{S}_{a b} \bar{\lambda}_{\dot{\alpha}}^{a} \bar{\lambda}_{\dot{\beta}}^{b} \varepsilon^{\dot{\alpha} \dot{\beta}}|0\rangle$ (where $\bar{\sigma}^{\mu v}=\frac{1}{4}\left(\bar{\sigma}^{\mu} \sigma^{v}-\bar{\sigma}^{v} \sigma^{\mu}\right)$, such that $\bar{\sigma}^{j 0}=\frac{1}{2} \tau_{j}$ as a $2 \times 2$ matrix with the first index up and the second down). Here $\mathscr{V}_{j}^{a}$ and $\mathscr{S}^{a b}=\mathscr{S}^{b a}$ are arbitrary and covariance allows us to write this as $\mathscr{V}_{j}^{a}=h_{1} \hat{c}_{j}^{a} / \hat{r}-h_{2} \hat{B}_{j}^{a} / \hat{r}^{2}+h_{3} \hat{c}_{j}^{b} \hat{c}_{k}^{b} \hat{c}_{k}^{a} / \hat{r}^{3}$ and $\mathscr{S}^{a b}=h_{4} \delta^{a b}+h_{5} \hat{c}_{j}^{a} \hat{c}_{j}^{b} / \hat{r}^{2}+h_{6} \hat{c}_{j}^{a} \hat{c}_{j}^{d} \hat{c}_{k}^{d} \hat{c}_{k}^{b} / \hat{r}^{4}$, where $h_{i}$ depend on $\hat{r}^{2}=\left(\hat{c}_{j}^{a}\right)^{2}, u=\hat{r}^{-4}\left(\hat{B}_{j}^{a}\right)^{2}$ and $v=\hat{r}^{-3} \operatorname{det} \hat{c}$. This gives a six dimensional matrix equation for the Hamiltonian which can be split into a "radial" and "angular" part $\left(\hat{H}_{f}=H_{f} / \hat{r}\right)$,

$$
H_{n^{\prime} n}^{p^{\prime} p}=\left\langle p^{\prime}, n^{\prime}|H| p, n\right\rangle=E_{p}^{\ell_{n}} \delta_{n n^{\prime}} \delta^{p p^{\prime}}+\left\langle p^{\prime}, \ell_{n^{\prime}}\left|\hat{r}^{4}\right| p, \ell_{n}\right\rangle\left\langle n^{\prime}\left|\frac{u}{2}\right| n\right\rangle+\left\langle p^{\prime}, \ell_{n^{\prime}}|\hat{r}| p, \ell_{n}\right\rangle\left\langle n^{\prime}\left|\hat{H}_{f}\right| n\right\rangle .
$$

Our basis explicitly diagonalizes the angular part ("spherical harmonics") in terms of invariant polynomials. In Fig. 2 the angular band structure is given by plotting $\left\langle n^{\prime}|u| n\right\rangle \neq 0$ or $\left\langle n^{\prime}\left|\hat{H}_{f}\right| n\right\rangle \neq 0$

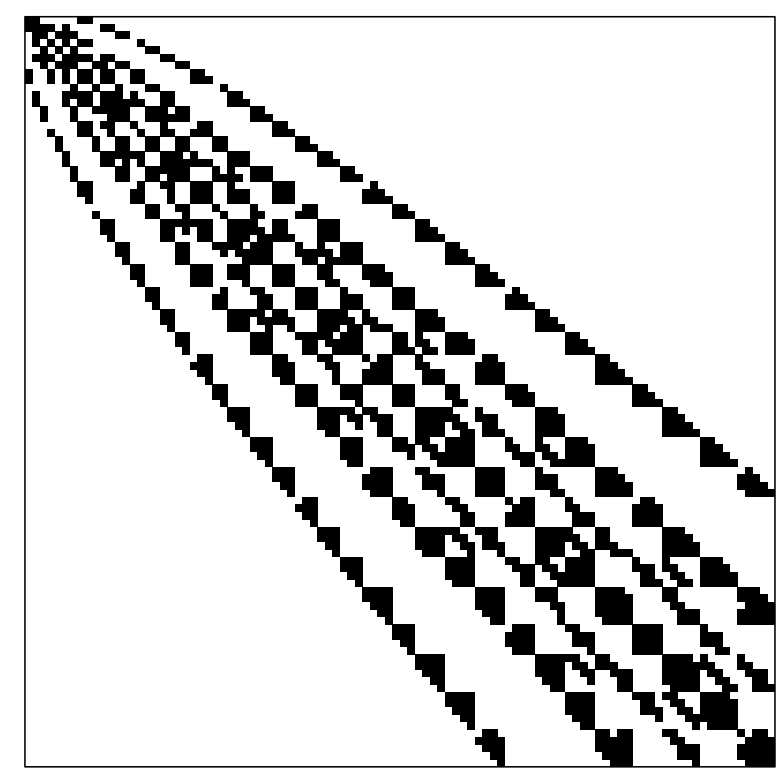

Figure 2: Band structure of the reduced Hamiltonian, for the first 100 states.

as black squares. The different bands can be traced to come from the selection rules $|\delta \ell|=0,2,4$ for the matrix elements of $u$, and $|\delta \ell|=1$ for the matrix elements of $\hat{H}_{f}$.

The radial wave function is $\phi_{p}^{\ell}(\hat{r})=C_{p}^{\ell} \hat{r}^{-3} j_{\ell}\left(k_{p}^{\ell} \hat{r}\right)$, with $E_{p}^{\ell}=\frac{1}{2}\left(k_{p}^{\ell}\right)^{2}$ and $\ell=2 L+3\left(j_{\ell}(z)\right.$ is the spherical Bessel function and $C_{p}^{\ell}$ is the normalization constant). It diagonalizes the kinetic term, $-\frac{1}{2} \hat{r}^{-8} \partial_{\hat{r}} \hat{r}^{8} \partial_{\hat{r}}+\hat{r}^{-2} L(2 L+7)=-\frac{1}{2} \hat{r}^{-3}\left[\hat{r}^{-2} \partial_{\hat{r}} \hat{r}^{2} \partial_{\hat{r}}+\frac{1}{2} \hat{r}^{-2} \ell(\ell+1)\right] \hat{r}^{3}$. To complete the construction of the basis, we need to address the question of boundary conditions (fixing the momenta $k_{p}^{\ell}$ ), so that the groundstate wave function becomes a constant, after projecting to the vacuum valley. The vacuum valley is characterized by those configurations for which $u=0$ (this implies $v=0$ ), and $\hat{r}$ measures the (three-dimensional) distance to the origin along this vacuum valley. 
The wave function can be decomposed as $\Psi=\sum_{n=0}^{\infty} \hat{r}^{-3} f_{n}(\hat{r}) \chi_{n}(u, v ; \hat{r})$, with $\chi_{n}(u, v ; \hat{r})$ normalized eigenfunctions, so that $\left\langle\Psi \mid \Psi^{\prime}\right\rangle=\sum_{n=0}^{\infty} \int 4 \pi \hat{r}^{2} d \hat{r} f_{n}^{*}(\hat{r}) f_{n}^{\prime}(\hat{r})$. This means that we have to impose $\partial_{\hat{r}} f_{0}(\hat{r})=0$ at the boundary of the fundamental domain, $\hat{r}=b \equiv \pi g^{-2 / 3}(L)$. This is equivalent to $\left\langle\chi_{0} \mid \partial_{\hat{r}}\left(\hat{r}^{3} \Psi\right)\right\rangle=0$, where the inner product is at fixed $\hat{r}$. That this boundary condition receives corrections is however an artifact of the truncation to the zero-momentum modes. If we take into account that in the full theory $\chi_{0}$ also involves the non-zero momentum modes, the (gauge) symmetry guarantees [10] that at the boundary of the fundamental domain $\partial_{\hat{r}} f_{0}(b)=0$, and this source of the breaking of supersymmetry is absent. Higher order terms in computing the effective Hamiltonian are required to deal with this [3]. We therefore impose $\partial_{\hat{r}}\left(\hat{r}^{3} \Psi(\hat{r})\right)=0$ at $\hat{r}=b \equiv \pi g^{-2 / 3}(L)$, knowing that it is only valid at small $g(L)$.

A direct measure for the failure of the Born-Oppenheimer approximation is given by $f^{2}(\hat{r})-$ $f_{0}^{2}(\hat{r}) \equiv \sum_{n=1}^{\infty} f_{n}^{2}(\hat{r})$, which as expected, deviates from zero when the gap is small, but also when we approach the boundary at $\hat{r}=b$ (due to the zero-momentum approximation). In Fig. 3 we show the results for $b=4.4,4.7$ and 5.0, to illustrate that this second deviation decreases with increasing $b$ (or decreasing coupling). This shows that the mismatch between the boundary condition and the truncation of the effective Hamiltonian does not affect the wave function in the neighborhood of the orbifold singularities, where the failure of the adiabatic approximation is non-perturbative.
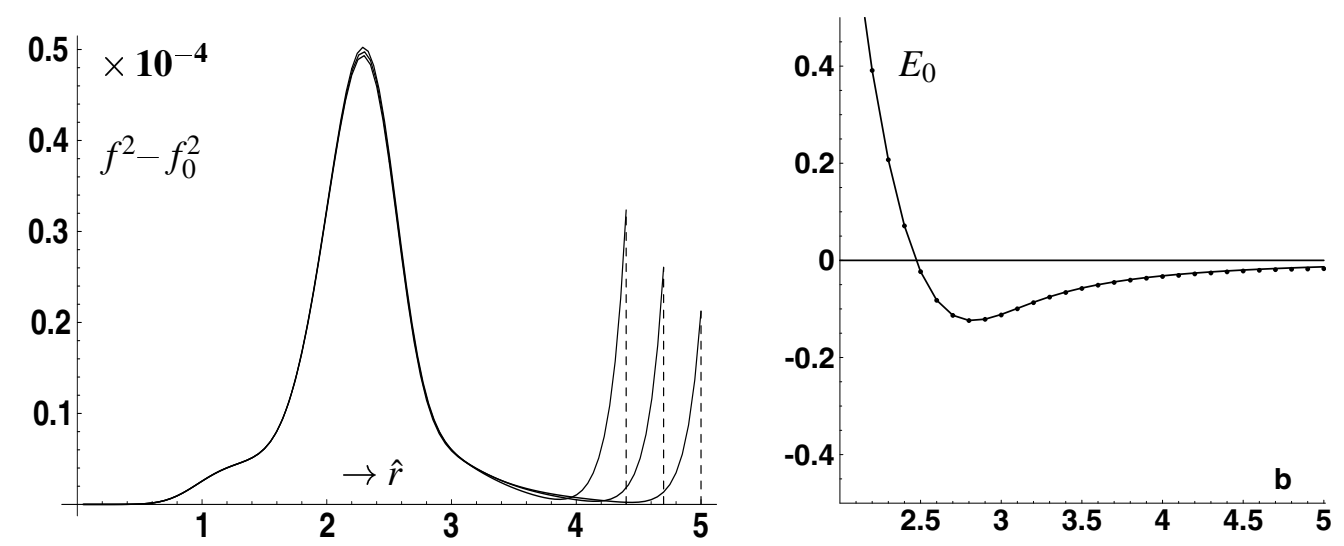

Figure 3: On the right is shown $f^{2}(\hat{r})-f_{0}^{2}(\hat{r})$ extracted from the groundstate wave function $\Psi_{0}$, satisfying the boundary condition $\partial_{\hat{r}}\left(\hat{r}^{3} \Psi_{0}\right)(b)=0$, for $b=4.4,4,7$ and 5.0. We normalized with respect to $b=5$, such that at $\hat{r}=2$ all $f^{2}$ agree. On the left is shown the groundstate energy itself, using up to 20 radial modes for each of the 420 harmonics together with the lower bound from Temple's inequality (indicated by the dots).

Finally Fig. 3 also shows the groundstate energy $E_{0}$ (the curve gives the upper and the dots the lower bound). To keep the size of the matrix manageable, the components of the eigenvectors are removed when they are in absolute value below a threshold, without significantly affecting the accuracy. This process of pruning is performed iteratively, increasing the number of radial modes per angular state. Together with Temple's inequality [10] this is extremely efficient to optimize the accuracy and achieve numerical control.

We have only considered $\mathrm{SU}(2)$ to illustrate how to go beyond the adiabatic approximation. Our analysis shows that, although the orbifold singularities are cause for concern, in the end they do not upset the result for the Witten index. For other groups this is much harder. Of course, a numerical analysis can never be entirely conclusive in deciding a theoretical issue 
that involves the counting of exact zero-energy states. The computer code is still available at www.lorentz.leidenuniv.nl/vanbaal/susyYM (partly they could be used for other studies [11]).

\section{Acknowledgments}

First I wish to thank Jean-François Mathiot for allowing me to speak on LC2008, particularly because it was on the Witten index. Apparently I could still present it, and ultimately write it down. Also thanks to Koenraad Schalm, who read the manuscript. But I must now also thank Margarita García Pérez and Tony González-Arroyo for inviting me to the Benasque Workshop in 25 February2 March 2007 and Christian Lang for iniviting me to the "Graduate Day" in Graz on 15 June 2007. Special thanks go to Simon Hands (and many other people) for letting me still participate in the Newton Institute programme "Strong Fields, Integrability and Strings", 19 August-6 October 2007 (while I was there, Bernard Piette invited me also for the "Classical Field Theory Workshop", which took place at Durham, 20-21 September 2007). I am also grateful for the invitation to the INT program "From Strings to Things" in Seattle, 5-17 May 2008, and espesially for the invitation to Galileo Galilei Institute for Theoretical Physics in Florence from 26 May-14 June 2008. Valya Zakharov and many other people have made this the best workshop I attended since my stroke, and I would like to thank especially Mithat Ürsal for taking me serious. Finally I thank Ayse Kizilersu and Tony Williams for inviting me to the "T(R)OPICAL QCD" workshop in Port Douglas, 27 July-1 August 2008. At all of these I gave seminars about Calorons, in one way or another.

\section{References}

[1] E. Witten, Nucl. Phys. B 202, 253 (1982).

[2] V. Novikov, M. Shifman, A. Vainshtein and V. Zakharov, Nucl. Phys. B 260, 157 (1985); M.A. Shifman and A.I. Vainshtein, Nucl. Phys. B 296, 445 (1988).

[3] P. van Baal, in: Michael Marinov Memorial Volume, eds. M. Olshanetsky and A. Vainshtein (World Scietific, Singapore, 2002), p.556 [hep-th/0112072].

[4] E. Witten, J. High Energy Phys. 02, 006 (1998) [hep-th/9712028].

[5] A. Keurentjes, A. Rosly and A.V. Smilga, Phys. Rev. D 58, 081701 (1998) [hep-th/9805183]; A. Keurentjes, J. High Energy Phys. 05, 001 (1999) [hep-th/9901154]; J. High Energy Phys. 05, 014 (1999) [hep-th/9902186]; Ph.D thesis (Leiden, June 2000) [hep-th/0007196]; V.G. Kac and A.V. Smilga, in: The many faces of the superworld: Yuri Golfand memorial volume, ed. M.A. Shifman (Worlf Scientific, Singapore, 2000), p.185 [hep-th/9902029].

[6] A. Borel, M. Friedman and J.W. Morgan, Memoirs of the American Math. Soc., Vol. 157, No. 747 (2002) [math.gr/9907007]; E. Witten, Adv. Theor. Math. Phys. 5, 841 (2002) [hep-th/0006010].

[7] G. 't Hooft, Nucl. Phys. B 153, 141 (1979).

[8] H. Itoyama and B. Razzaghe-Ashrafi, Nucl. Phys. B 354, 85 (1991); M.B. Halpern and C. Schwartz, Int. J. Mod. Phys. A 13, 4367 (1998) [hep-th/9712133].

[9] M. Lüscher, Nucl. Phys. B 219, 233 (1983).

[10] J. Koller and P. van Baal, Nucl. Phys. B 302, 1 (1988).

[11] J. Wosiek, Acta Phys. Pol. B 34, 5103 (2003) [hep-th/0309174]; M. Campostrini and J. Wosiek, Nucl. Phys. B 703, 454 (2004) [hep-th/0407021]. 